\title{
Application Status and Research of Instrumentation Device in Solar Thermal Power Generation Industry
}

\author{
Xiaojun XIE, Hang XI, Qiong WU' ${ }^{1}$, Ruigang ZHANG, Bo YANG, Zengbo LIU \\ Xi'an Thermal Power Research Institute Co., Ltd., Xi'an 710054, China
}

\begin{abstract}
The solar thermal power generation system adopts a dual-axis timely tracking instrument device, which realizes that the sunlight and the central axis of the heliostat instrument device are kept parallel, and greatly improves the utilization efficiency of the light source and the power generation efficiency. At the same time, the study of instrumentation selection in the solar thermal power generation industry cannot be ignored, which can guarantee the normal operation and basic work quality of solar thermal power projects. Therefore, based on instrumentation devices in the solar thermal power generation industry, this article explores the drawbacks of instrumentation devices in the application, and puts forward several research ideas for the drawbacks. Finally, by taking the tower-type solar thermal power generation instrument device as an example.
\end{abstract}

Keywords. solar thermal power generation system, instrumentation selection, instrument device

\section{Introduction}

At present, with the trend of global economic development, the content of traditional energy reserves has been decreasing year by year, coupled with the increasing environmental pollution problem, the research and application of new green energy becomes very critical. From December 16 to 18, 2020, the Central Work and Economic Conference clearly pointed out that in order to achieve carbon peak and carbon neutral in the future, accelerating the adjustment and optimization of industrial structure and energy structure, promote coal consumption to peak as soon as possible, vigorously develop new energy, accelerate the construction of the national energy use right and carbon emission right trading market, and improve the double control of energy consumption [1-3]. New energy power generation undoubtedly ushered in an excellent opportunity for development. Solar energy is an inexhaustible source of energy, the use of solar thermal

${ }^{1}$ Corresponding author: Qiong Wu, Xi'an Thermal Power Research Institute Co., Ltd., Xi'an 710054, China; E-mail: wuqiong.0702@163.com. 
power can avoid the reliance on coal, oil, natural gas and other non-renewable energy. In 2019 , the installed capacity of grid-connected solar power generation was 204.68 million kilowatts, up 17.2 percent from 2018. By the end of 2019, Qinghai Gonghe 50MW molten salt tower solar thermal power generation project (Figure 1), Golmud 50MW molten salt tower type solar thermal power generation project (Figure 2), Hami 50MW molten salt tower type solar thermal power generation project (Figure 3) and Dunhuang $50 \mathrm{MW}$ molten salt linear fresnel solar thermal power generation project (Figure 4) have all been successfully connected to the grid [4].

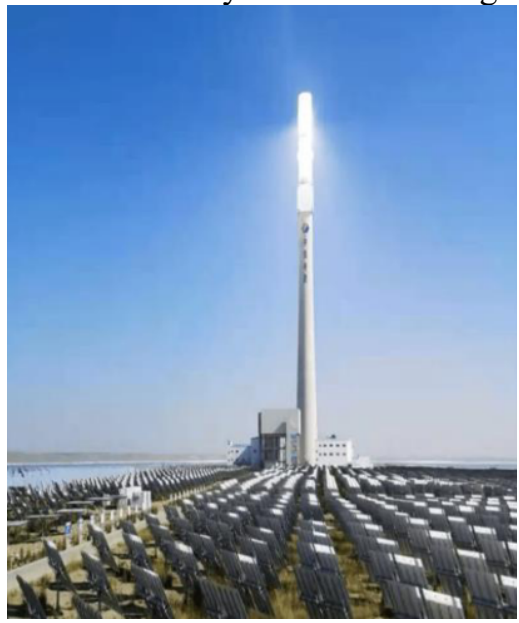

Figure 1. Qinghai Gonghe 50MW molten salt tower solar thermal power generation project.

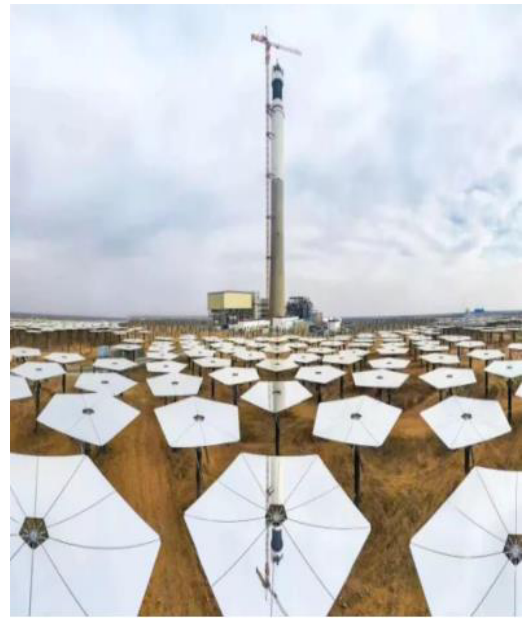

Figure 3. Hami 50MW molten salt tower solar thermal power generation project.

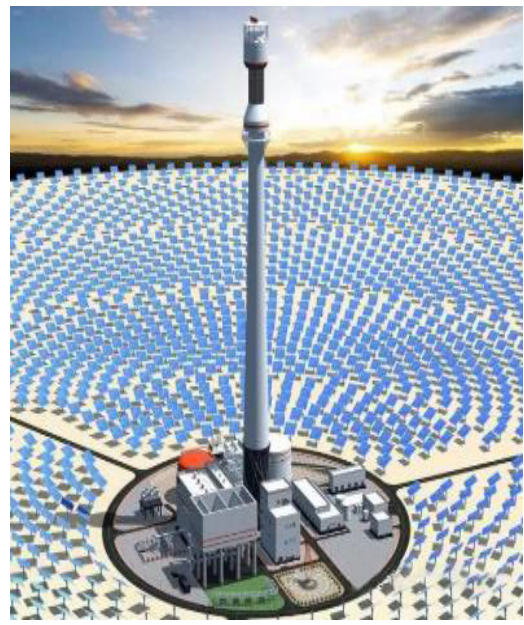

Figure 2. Golmud 50MW molten salt tower solar thermal power generation project.

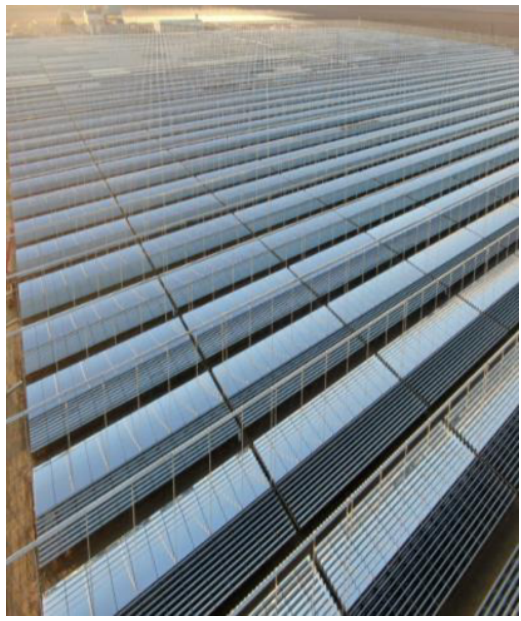

Figure 4. Dunhuang 50MW molten salt linear fresnel solar thermal power generation.

All of the above indicates that the solar thermal power generation industry, as the foundation of the national power competition in the future, has broad prospects for development. However, there are still some typical quality problems in the current manufacturing and operation stage of the solar thermal power generation industry. Mainly includes the reflectivity of the mirror is unqualified, electric push rod tracking function day by day is failed, steel structure galvanizing treatment is unqualified, the 
quality of storage tank steel plate is unqualified, welding quality of molten salt storage tank is unqualified, the high dynamic accuracy of the high pressure rotor of the steam turbine is unqualified, system instrumentation accuracy is unqualified, etc. The above-mentioned typical quality problems are caused by many factors, including design factors, manufacturing factors, installation factors and operation and maintenance factors. The utilization efficiency and generation efficiency of the light source in the solar thermal power generation system cannot be separated from the instrument device. So, it is necessary to study the selection and application of the instrument device. In addition, it is reported that Sun Jiong from Ningbo Ozaki has designed and manufactured the first production line of " armored thermocouple cable and armored platinum resistance cable" in China [5]. The production line successfully shortens the gap between domestic and foreign thermal instrument manufacturing field, and then makes an important contribution to the development of innovative high quality equipment localization. This will also promote the high level of solar thermal instrument manufacturing, and to help us selection and application.

\section{Disadvantages of Instrumentation in the Application of Solar Thermal Power}

\section{Generation Industry}

\subsection{Poor Application Performance of Instrumentation Device}

The selection and application of instrumentation devices in the solar thermal power generation industry play an important role. At present, the design of the instrumentation device is customized in advance according to the needs. The entire design process is completely in accordance with the rules and regulations of the scientific instruction manual to ensure the stability of the instrumentation device [6]. Since the users of the instrumentation device do not pay more attention to the stability of the instrument in the daily use, the poor arrangement of the instrument use position greatly limits the stability of the instrumentation device. In addition, the operator's inattention to the instrument and insufficient understanding of the technology of the instrument device result in the poor stability of the application of the instrument device in the solar thermal power generation industry.

\subsection{Insufficient Maintenance of Instrumentation Device}

Any instrument device can not be separated from the daily maintenance especially in the solar thermal power generation industry that requires high accuracy. If you do not pay enough attention to the daily maintenance of the instrumentation device and ignore some common small problems. For example, the thermal power generation industry causes long-term chronic corrosion of thermal equipment due to water vapor problem. In a short time, there will be no accident problem in general, but in the long run, it will cause equipment abnormalities. However, without daily maintenance, it is impossible to detect the thermal equipment and reflect the stability of the thermal equipment in real time for the detection of online detection instruments. Therefore, the application of instrument devices in solar thermal power generation projects needs to pay special attention to routine maintenance [7-8]. 


\subsection{Lack of Advancement and Creativity}

At present, the manufacturing technology and application function of the instruments used in the solar thermal power generation industry are not advanced enough in our country. Few instrumentation device are developed and applied customized according to the application objects, and there are problems such as the gap between instrument accuracy and foreign products. The above problems seriously restrict the promotion and application of high quality instrumentation products in the solar thermal power generation industry.

\section{Application Ideas of Instrumentation Device in the Solar Thermal Power Generation Industry}

\subsection{Improve the Stability of the Instrumentation Device}

In order to improve the stability of the instrumentation, the solar thermal power generation industry needs to adopt a targeted strategy to use different instrumentation device for different power generation projects, while ensuring that the technical standard and specifications of the instrumentation device are the same. In addition, the standard operation and use position of the instrumentation device can also improve the use of stability. In this way, the stability of the maintenance and management of the instrumentation device are ensured, and then the work efficiency of the project construction is improved.

\subsection{Pay Attention to the Daily Maintenance of Instrumentation Device}

The solar thermal power generation industry would be focused on the importance of online instrumentation applications. The first is to increase the professional team for instrumentation maintenance; the second is to appropriately increase the scientific research funding for instrumentation for the development of high-precision, high-efficiency instrumentation and procurement of parts and replacement and maintenance of parts. In addition, the solar thermal power generation industry needs to strengthen the supervision and management of the instrumentation device, and timely prevent the abnormal application of the online instrumentation device caused by the lack of responsibility of the maintenance personnel and the inadequate maintenance. In terms of instrumentation maintenance, both companies and individuals need to regularly participate in internal communication work, strengthen communication and exchanges between various departments of solar thermal power generation, and improve the application level of instrumentation device [9-10].

\subsection{Regularly Calibrate and Overhaul Instrumentation Device}

The use accuracy of the instrument directly affects the tracking accuracy, which in turn leads to the reduction of the utilization efficiency and power generation efficiency of the light source of the solar thermal power generation station. It is very necessary to calibrate and overhaul the accuracy of the instrument on a regular basis. Although this work will increase part of the investment, it is very important for the accurate measurement and 
normal use of instruments and meters. It can not only ensure the normal operation of the system, but also avoid unnecessary power loss and increase power generation revenue.

\section{Application of Instrumentation in the Solar Thermal Power Generation}

\section{Industry-Taking Tower Solar Thermal Power Instrumentation Device as An Example}

\subsection{The Structure of Tower Solar Thermal Power Generation Station and Instrumentation}

Tower-type solar thermal power generation system is also called centralized solar thermal power generation system. The basic form of the tower-type solar thermal power generation system is to use a group of heliostats that independently track the sun to gather sunlight on the receiver on the top of the fixed tower to generate high temperature, heat the working fluid to generate superheated steam or high-temperature gas, and drive the steam turbine or a gas turbine generator to convert solar energy into electrical energy [11]. Tower-type solar thermal power generation is one of the applications of many projects, and its system includes heliostat array, heat collection system, heat storage system, power generation system and control system. The schematic diagram of the tower-type solar thermal power generation system is shown in the figure 5:

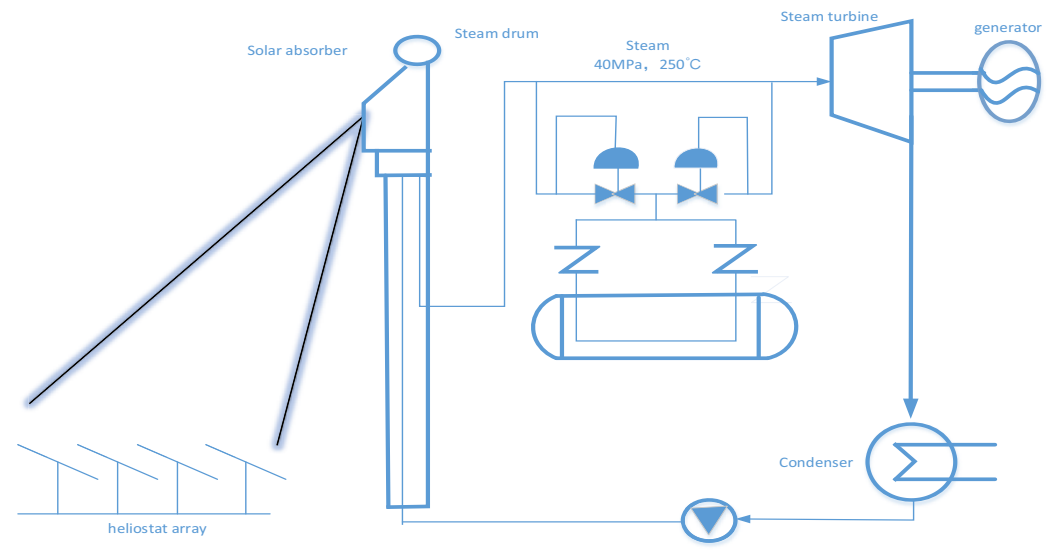

Figure 5. Schematic diagram of tower-type solar thermal power station.

In order to ensure that the system can operate efficiently and accurately, it is necessary to configure relevant instrumentation devices to improve the overall efficiency of the system and the efficiency of power generation. The instrumentation devices involved include heliostats, heat-absorbing heat accumulators, etc.

\subsection{Configuration of Tower-Type Solar Thermal Power Generation Instrument}

The tower-type solar thermal power station uses the solar energy collection mirror field instrument control system, and under the configuration of wireless instrument and network technology, it can effectively save a large number of control cables and debugging functions, and maximize the overall efficiency of the engineering 
infrastructure [12]. The configuration of the tower-type solar thermal power generation instrument process system is as follows: through the placement of the heat-absorbing heat accumulator with high-purity graphite, and the placement of the matching heliostat instrument under each tower to achieve the concentration of sunlight reflection, and converge the concentrated solar beam to the inside of the opening. Among them, the mirror field instrument can use wireless instrument or network technology to control the service in the application configuration [13]. The tower-type solar thermal power generation devices system includes the following instrument control devices. One is that the heat-absorbing and heat-storing instrument device realizes the treatment of the temperature of the medium. The heat-absorbing and heat-storage instrument device needs to work in a suitable temperature range. Once the temperature is too high, it will restrict the safe operation of the system, such as causing the breakdown of the medium layer and the damage of the instrument device. Once the temperature is too low, it will not be able to provide the best power generation technical support for the turbo-generator at the appropriate temperature. The second is the configuration of the steam temperature control instrument for the heat-absorbing accumulator. Because the temperature of the internal heat storage medium will gradually decrease with the steam process (the transfer of heat energy), resulting in a decrease in the temperature difference between the medium and the feed water, it is necessary to control the flow of the feed water through the adjustment function. Among them, the feed water flow rate and the heat storage medium should maintain a proportional relationship, so as to meet the operation requirements of the steam turbine. The third is the configuration of the heliostat control instrument. This configuration is related to the complexity of investment and technology, and is designed reasonably according to actual requirements.

\subsection{Network Device Selection of Tower-Type Solar Thermal Power Generation Devices}

According to the characteristics of local monitoring equipment, the heliostat instrument of tower-type solar thermal power generation instrument is configured into corresponding wireless multi-combination signal converter, or the heliostat local controller is used [14]. Specifically, the application of local controller of heliostat instrument focuses on the reasonable calculation and state monitoring of heliostat driving motor position instructions. If the heliostat instrument has no local control device, the design of the system needs to be completed under the mirror field control server. In addition, a wireless multi-function node is arranged at the heat absorption and accumulator on the top of the solar tower, and communication is realized between the corresponding heliostat instrument and the transmitter instrument. The power supply mode of wireless instrument device usually adopts the charging mode of local power supply. Since the driving mechanism needs power supply support, only the power cord can be connected, so the corresponding parameters (refresh power, transmit power) are determined according to the actual situation [15]. Wireless transmitter in the data refresh speed can reach 1 second, but also according to the actual situation, reasonable setting of the refresh rate. For example, the wireless transmitter of the heliostat instrument device can collect the angle position in real time, and obtain the altitude angle and azimuth angle of the sun with the help of the meteorological observation station, so as to compare the data with the actual altitude angle and azimuth of the sun, so as to reduce the errors in the long-term operation of the heliostat instrument. 


\section{Conclusion}

This paper mainly takes the tower solar thermal power generation instrument device as an example, from the system introduction, instrument device configuration, device selection of three aspects of the instrument device configuration and network configuration of the comprehensive application. A new application idea is put forward for the application of instruments in the solar thermal power generation industry. In view of the application requirements of this instrument system in solar thermal power generation industry, the selection of heliostat instrument and device is analyzed and studied.

\section{Acknowledgements}

This paper was supported by the "Wind-photovoltaic-water coordinating optimization technology research" of science and technology project of Huaneng Group Headquarters (HNKJ18-H35).

\section{References}

[1] Yuan WD. Current development and prospect of solar-thermal power generation in china and abroad. Power \& Energy, 2015(4):487-490.

[2] Wei ZK. Current status and perspective for solar power tower technology. Encyclopedia Forum, 2019(11):528-529.

[3] Huang W. Running state detection of heliostat for tower power station [D].Nanjing Normal University,2016.

[4] Solarbe. In 2019, the installed capacity of global solar thermal power generation increased to 6451MW, China contributed $52.41 \%$ to the new capacity [EB/OL] . https://www.sohu.com/a/365430945_418320, 2020-1-8.

[5] Association for Science and Technology Innovation. Ningbo Aoqi: Drive China's Thermal Instrument Manufacturing Technology to the Forefront of the World [EB/OL]. http://www.cspplaza.com/article-11697-1.html, 2018-3-14.

[6] Yuan H, Li F, Wang CX. Reason analysis on measuring error of on-line chemical instrument in thermal power plant and improvement measures. Shanxi Electric Power, 2019(5):49-51.

[7] Zhang YJ. Common problems and countermeasures in quality control of power engineering construction projects. Science and Wealth, 2020(06):61-61.

[8] Wang K, Ding L, Zang PW, Sun DK. Analysis on installation of molted salt absorber and selection of related equipment for tower solar thermal power station. Technology and Market, 2020(7):53-54, 58.

[9] Zhao CY. Discussion on the importance of measurement and detection of instruments and meters. Technology and Information, 2018, (027):121-123.

[10]Li PC. Optimization and experimental investigation of cascade Rankine cycle-based solar thermal power generation system [D]. University of Science and Technology of China, 2016.

[11]Song LN. Design and implementation of heliostat control system [D].University of Electronic Science and Technology, 2016.

[12]An RC. Design and selection of electric heater for molten salt storage tanks in solar thermal power plants. China Resources Comprehensive Utilization, 2019, 037(011):59-62.

[13]Wang YS. Research on design of molten salt heat storage tank [D]. Shenyang Institute of Engineering, 2019.

[14]Li CY, Hu LG, XYU Tong-she. Wireless instrument and network configuration of tower type csp power plant. Electric Power Survey \& Design, 2015(2):78-80.

[15]Xu FB. Research on structure selection and mechanical property of heliostat supporting structure of dual-aixs tracking CSP system [D]. Beijing: Beijing Jiaotong University, 2012. 